\title{
Asymmetric Procyclicality of Chinese Banking and the Countercyclical Buffer of Basel III
}

\author{
Yufeng $\mathrm{Li}^{1}$ and Zhongfei $\mathrm{Li}^{2}$ \\ ${ }^{1}$ School of Mathematics and Statistics, Lanzhou University, Lanzhou 730000, China \\ ${ }^{2}$ Sun Yat-sen Business School, Sun Yat-sen University, Guangzhou 510275, China \\ Correspondence should be addressed to Zhongfei Li; lnslzf@mail.sysu.edu.cn
}

Received 22 October 2014; Revised 15 December 2014; Accepted 17 December 2014

Academic Editor: Carlo Piccardi

Copyright (c) $2015 \mathrm{Y}$. Li and Z. Li. This is an open access article distributed under the Creative Commons Attribution License, which permits unrestricted use, distribution, and reproduction in any medium, provided the original work is properly cited.

\begin{abstract}
Since the global financial crisis of 2007-2008, the importance of the procyclicality in the banking sector has been highlighted. One of the Basel III objectives is to promote countercyclical buffers and reduce procyclicality. We apply time-varying copula combined with GARCH model to test the existence of asymmetric procyclicality of Chinese banking. The results show that the procyclicality of Chinese banking is asymmetric, where the dependence between loan and economy growth is more correlated during the decline stage than the rise stage of economy. Based on this asymmetry, we suggest that the authority can use high frequent index for signalling the start point of releasing countercyclical buffer and accelerate the releasing pace to avoid the supply of credit being constrained by regulatory capital requirements in downturns.
\end{abstract}

\section{Introduction}

The interaction of macroeconomy and financial sector variables has long been an object of interest to economists. Keynes [1] argued that informational asymmetry was an inherent problem in the functioning of the financial markets and this uncertainty fluctuates with the real economy. Bernanke and Blinder [2] and Kiyotaki and Moore [3] argued that even relatively small shocks to the economy could be amplified by endogenous changes in the credit market conditions, and this phenomenon leads to the propagation of the business cycle by introducing the financial accelerator model.

In particular, since the global financial crisis of 20072008 , the importance of the procyclicality in the banking sector has been highlighted. Ideally, the banking system should provide a safety net to enterprises and households to mitigate economic volatility. However, the financial crisis and the decline of output can be observed at the same time and they even intensify each other. During this crisis, the major global banking institutions reported cumulative losses and write-downs of $\$ 1306$ billion by April 2010, while world GDP contracted by 1.6 percent between mid-2008 and mid-2009 (see [4]).
There is a considerable literature, both theoretical and empirical, which indicates that bank loan growth is cyclical to the changing macroeconomy activity. Loan supply is determined by bank capital and credit standards, which are dependent on the economic activity cycle. Jokipii and Milne [5] argued for the presence of a negative relationship between European banks' capital buffers and the GDP over the economic cycle. Puri et al. [6] reported that Changes in a bank's capital might induce changes in its loan supply. Berger et al. [7] argued that US banks changed their bank credit standards depending on the current phase of the economic cycle. ECB [8] argued that the high GDP growth was accompanied by softer bank credit standards, increased loan amounts, and maturities. Bank of Greece [9] indicated that the decline in GDP during the last quarter of 2009 was accompanied by more stringent bank credit standards and higher rejection rates for small and medium enterprises.

Due to this procyclicality of loan growth behavior, banks usually provide loans to those risky projects with marginally positive or even a negative net present value in the upturns, while they do the opposite in the downturns. Thus, the banking system, rather than compensating for swings in the economic activity over the cycle, makes them even more 
intense. The procyclicality of loan growth has transformed banks from mitigation mechanisms to amplifiers of changes in economic activity, potentially affecting financial stability and economic growth [10].

By the end of 2008, the G-20 agreed that it was important "to address the issue of procyclicality in financial markets regulations and supervisory systems." They called upon the IMF, the Financial Stability Board, and the Basel Committee to identify ways to alleviate it. Now several suggestions have been forwarded to attenuate procyclicality, in the form of rules and discretion. Some of the suggestions have been adopted under the Basel III framework, which explicitly addresses the issue of procyclicality.

Basel III reforms are meant to strengthen the banking sector and raise the resilience of individual banking institution to the period of stress. One of the Basel III objectives is to promote countercyclical buffers and reduce procyclicality. The objective of the countercyclical buffer is to ensure the ability of the whole banking sector to provide loans to the economy during recessions and to protect banks from taking significant risks during periods of excessive credit growth. Banks will be subject to a countercyclical buffer that varies between $0 \%$ and $2.5 \%$ to total risk weighted assets [11]. It will be built up when excess aggregate credit growth is judged to be associated with a buildup of system-wide risk to ensure that the banking system has a buffer of capital to protect it against future potential losses. Also, the buffer will be released in times of stress to reduce the risk of the supply of credit being constrained by regulatory capital requirements. The commission suggested that the credit/GDP guide was a useful common reference point in taking buffer decisions. According to the guide, the size of the buffer (in percent of risk-weighted assets) is 0 when credit/GDP gap is below the lower threshold 2\%, while it reaches its maximum level $2.5 \%$ when the gap exceeds the upper threshold $10 \%$. When the credit-to-GDP gap is between $2 \%$ and $10 \%$, the buffer add-on will vary linearly between $0 \%$ and $2.5 \%$ [12]. A lot of research has demonstrated that credit-to-GDP gap performed well for taking buffer decision in the build-up phase. Drehmann et al. [13] indicated that the credit-to-GDP ratio tended to rise smoothly well above trend before the most serious episodes. Unfortunately, related research indicated that the variable credit-to-GDP gap was not an ideal indicator variable for signalling the release phase.

Naturally, we suspect that the asymmetry dependence on loan growth and economy activity may be the reason of the above disfunction of taking buffer decisions in release phase. Actually, the asymmetric procyclicality of credit risk has been verified, which indicated that the effect of the business cycle on credit risk is more pronounced during downturns by Marcucci and Quagliariello [14]. However, the asymmetric procyclicality of loan growth has been paid little attention.

Copula-based models provide a great deal of flexibility in modeling multivariate distributions, allowing the researcher to specify the models for the marginal distributions separately from the dependence structure (copula) that links them to form a joint distribution [15]. It has become a popular concept for measuring the stochastic dependence between random variables. Copulas can deal with complex multivariate correlation structure such as nonlinearity and asymmetry in that they provide a way of isolating the description of the dependence structure from the marginal distribution function. Mendes [16] investigated the asymmetric extreme interdependence of emerging equity markets applying copula approach and indicated that the dependence was typically stronger during bear markets. Durante and Jaworski [17] investigated the spatial contagion between financial markets based on a threshold copula approach. Recently, copula theory has been extended to the conditional case, allowing the use of copulas to model dynamic structures. Patton [18] extended the theory of (unconditional) copulas to the conditional case, which allow us to use copula theory in the analysis of time-varying conditional dependence, and examined the dependence structure of daily Deutsche markUS dollar (DM-USD) and Japanese yen-US dollar (Yen-USD) exchange rates.

In this paper, we apply time-varying copula combined with GARCH model to analyze the dependence of banking loan and macroeconomy growth in China. We mainly test the existence of asymmetric procyclicality of Chinese banking, which can provide beneficial suggestion for the implementation of the countercyclical buffer policy. The remainder of the paper is structured as follows. In Section 2, the copulas employed in this paper are described. Section 3 contains the data description, the data processing, and the empirical results. In Section 4, we analyze the reason of asymmetric procyclicality and its implications for financial controlling and regulatory. Finally, the main conclusions are summarized in Section 5.

\section{Methodology}

We use a time-varying copula approach to examine the dependence structure between banking loan and economy growth over time. We begin with modeling the margin of each time series by fitting appropriate ARMA-GARCH specifications to the data and extracting the standardized residuals. We then apply the empirical cumulative distribution function (ECDF) to obtain approximate i.i.d. (independently and identically distributed) Unif $(0,1)$ series that are suitable for further statistical analysis in copulas. At last, we estimate the static and time-varying parameters of copulas.

2.1. Models for Marginal Distributions. To model the margin of return series, we combine an $\operatorname{ARMA}(m, n)$ process with a standard GARCH $(p, q)$ model. This combination can successfully characterize some important stylized facts of time series such as volatility clustering and time-varying volatility. Given a time series $y_{t}$, an $\operatorname{ARMA}(m, n)-\mathrm{GARCH}$ $(p, q)$ model can be written as (see [19])

$$
\begin{gathered}
y_{t}=\mu+\sum_{i=1}^{m} a_{i} y_{t-i}+\sum_{j=1}^{n} b_{j} \varepsilon_{t-j}+\varepsilon_{t}, \\
\varepsilon_{t}=\sigma_{t} z_{t}, \\
\sigma_{t}^{2}=\omega_{0}+\sum_{i=1}^{p} \alpha_{i} \sigma_{t-i}^{2}+\sum_{j=1}^{q} \beta_{j} \varepsilon_{t-j}^{2},
\end{gathered}
$$


where $\omega_{0}>0, \alpha \geq 0, \beta \geq 0, \mu$ is a constant term of the conditional mean equation, $z_{t}$ is a sequence of i.i.d. random variables with zero mean and unit variance, and $\sigma_{t}^{2}$ denotes the conditional variance of return series at time $t$, which depends on both past return innovations $\varepsilon_{t-i}$ and past conditional variances $\sigma_{t-i}^{2}$. Usually, random variable $z_{t}$ is assumed to be a standard normal distribution, standardized Student's $t$ distribution, or standardized GED (generalized error distribution).

2.2. Definitions and Properties of Copula Functions. The introduction of copulas can be traced back to the statistician Sklar [20]. He proposed the famous Sklar theorem, which shows that any $n$-dimensional joint distribution function may be decomposed into its $n$ marginal distributions and a copula, which completely describes the dependence between the $n$ variables. Sklar [20] also gave the definition of "copula" as follows.

Definition 1. A two-dimensional copula is a function $C$ : $[0,1]^{2} \rightarrow[0,1]$ that has the following properties:

(i) $C(u, v)$ is increasing in $u$ and $v$;

(ii) $C(0, v)=C(u, 0)=0 ; C(1, v)=v ; C(u, 1)=u$;

(iii) for every $u_{1}, u_{2}, v_{1}, v_{2} \in[0,1]$ such that $u_{1}<u_{2}$ and $v<v_{2}$, we have $C\left(u_{2}, v_{2}\right)-C\left(u_{2}, v_{1}\right)-C\left(u_{1}, v_{2}\right)+$ $C\left(u_{1}, v_{1}\right) \geq 0$.

Theorem 2 (Sklar's theorem (see [20])). Let $H$ be a $n$ dimensional distribution function with marginals $F_{i}\left(x_{i}\right)$ with $i=1,2, \ldots, n$. Then, there exists a copula $C$ such that, for random variables $X_{i}$, one has

$$
H\left(x_{1}, x_{2}, \ldots, x_{n}\right)=C\left(F_{1}\left(x_{1}\right), F_{2}\left(x_{2}\right), \ldots, F_{n}\left(x_{n}\right)\right) .
$$

If $F_{i}\left(x_{i}\right)$ are continuous for all $i=1,2, \ldots, n$, then $C$ is unique. Conversely if $C$ is a copula and $F_{1}, F_{2}, \ldots, F_{n}$ are distribution functions, then the function $H$ defined above is a joint distribution with margins $F_{1}, F_{2}, \ldots, F_{n}$.

Patton [18] extended the theory of (unconditional) copulas to the conditional case, which allows us to use copula theory in the analysis of time-varying conditional dependence. He gave the definition of conditional copula as follows.

Definition 3. The conditional copula of $(X, Y) \mid W=w$ is the conditional joint distribution function of $U \equiv F_{X \mid W}(X \mid W)$ and $V \equiv F_{Y \mid W}(Y \mid W)$ given $W=w$, where $X \mid W=w \sim$ $F_{X \mid W}(\cdot \mid w)$ and $Y \mid W=w \sim F_{Y \mid W}(\cdot \mid w)$.

Theorem 4. Let $F_{X Y \mid W}(\cdot \mid w)$ be the joint conditional distribution of $(X, Y) \mid W=w$. Assume that $F_{X \mid W}(\cdot \mid w)$ and $F_{Y \mid W}(\cdot \mid w)$ are continuous in $x$ and $y$ for all $w \in W$. Then there exists a unique conditional copula $C(\cdot \mid w)$ such that

$$
F_{X Y \mid W}(x, y \mid w)=C\left(F_{X \mid W}(x \mid w), F_{Y \mid W}(y \mid w) \mid w\right) \text {. }
$$

2.3. Dependence Relation. Rank correlation concentrates on modeling the rankings of given observed data rather than on the actual values of the data themselves. There are two well-established measures of rank correlation, which are Spearman's rho and Kendall's tau.

Let $X$ and $Y$ be continuous random variables whose copula is $C$. Spearman's rho for $X$ and $Y$ is given by

$$
\rho_{X, Y}=12 \iint_{I^{2}} C(u, v) d u d v-3
$$

Kendall's tau for $X$ and $Y$ is given by

$$
\tau_{X, Y}=4 \iint_{I^{2}} C(u, v) d C(u, v)-1 .
$$

Tail dependence is applied to measure the dependence between the extreme values of random variables for copula models. Informally, it measures the probability that we will observe an extremely large positive (negative) realization of one variable, given that the other variable also took on an extremely large positive (negative) value. The definition of tail dependence is given by Nelsen [21]. Let $X$ and $Y$ be continuous random variables with distribution functions $F$ and $G$, respectively. The upper tail dependence coefficient between $X$ and $Y$ is defined as

$$
\lambda_{U}=\lim _{t \rightarrow 1^{-1}} P\left[Y>G^{-1}(t) \mid X>F^{-1}(t)\right]
$$

if this limit exists. The same concept is applied to define the lower tail dependence coefficient as

$$
\lambda_{L}=\lim _{t \rightarrow 0^{+}} P\left[Y \leq G^{-1}(t) \mid X \leq F^{-1}(t)\right]
$$

if this limit exists. These parameters are nonparametric and depend only on the copula of $X$ and $Y$. The following formulas give the relationship between tail dependence parameters with copula functions:

$$
\lambda_{U}=2-\lim _{t \rightarrow 1^{-}} \frac{1-C(t, t)}{1-t}, \quad \lambda_{L}=\lim _{t \rightarrow 0^{+}} \frac{C(t, t)}{t} .
$$

The measure $\lambda_{U}$ quantifies the amount of extremal dependence within the class of asymptotically dependent distributions. It provides a natural way for ordering copulas. If $\lambda_{U} \epsilon$ $(0,1]$, then $X$ and $Y$ are said to be asymptotically dependent in the upper tail. If $\lambda_{U}=0$, then $X$ and $Y$ are said to be asymptotically independent in the upper tail. It is similar for the lower tail dependence parameter.

2.4. Two Copulas. We will specify and estimate two alternative copulas, the "symmetrized Joe-Clayton" (SJC) copula and the Gaussian copula, both with and without time variation. We will assume that the functional form of the copula remains fixed over the sample whereas the parameters vary according to some evolution equation. 
The two-dimensional Gaussian copula is

$$
\begin{aligned}
& C_{G}(u, v ; \theta) \\
&= \Phi_{G}\left(\Phi^{-1}(u), \Phi^{-1}(v) ; \theta\right) \\
&=\int_{-\infty}^{\Phi^{-1}(u)} \int_{-\infty}^{\Phi^{-1}(v)} \frac{1}{2 \pi \sqrt{\left(1-\theta^{2}\right)}} \\
& \cdot \exp \left\{\frac{-\left(x_{1}^{2}-2 \theta x_{1} x_{2}+x_{2}^{2}\right)}{2\left(1-\theta^{2}\right)}\right\} d x_{1} d x_{2} .
\end{aligned}
$$

For the static Gaussian copula, the parameter $\theta$ is assumed to be constant over time. For the time-varying Gaussian copula, the correlation parameter $\theta_{t}$ is assumed to be evolving through time. In this paper, we assume that the time-varying correlation matrix

$$
R_{t}=\left[\begin{array}{cc}
1 & \theta_{t} \\
\theta_{t} & 1
\end{array}\right]
$$

complies with a DCC $(1,1)$ process (see [22]):

$$
\begin{gathered}
R_{t}=\widetilde{Q}_{t}^{-1} Q_{t} \widetilde{Q}_{t}^{-1}, \\
Q_{t}=(1-\alpha-\beta) \cdot \bar{Q}+\alpha \cdot \varepsilon_{t-1} \varepsilon_{t-1}^{\prime}+\beta \cdot Q_{t-1},
\end{gathered}
$$

where $\bar{Q}$ is the sample covariance of $\varepsilon_{t}$ and $\widetilde{Q}_{t}$ is a square $p \times p$ matrix with zeros as off-diagonal elements and diagonal elements as the square root of those of $Q_{t}$. The parameter constraints for the DCC are the same for the univariate $\operatorname{GARCH}(1,1)$ models: $\alpha+\beta<1, \alpha, \beta \in(0,1)$.

The distribution of the SJC copula is derived from the JoeClayton copula by Patton [18]:

$$
\begin{aligned}
C_{\mathrm{SJC}}\left(u, v \mid \tau^{U}, \tau^{L}\right) \\
=\frac{1}{2}\left(C_{\mathrm{JC}}\left(u, v \mid \tau^{U}, \tau^{L}\right)+C_{\mathrm{JC}}\left(1-u, 1-v \mid \tau^{U}, \tau^{L}\right)\right. \\
\quad+u+v-1),
\end{aligned}
$$

where $\tau^{U}$ is the upper tail dependence coefficient and $\tau^{L}$ is the lower tail dependence coefficient. The Joe-Clayton copula $C_{\mathrm{JC}}$ is defined as follows:

$$
\begin{aligned}
C_{\mathrm{JC}}\left(u, v \mid \tau^{U}, \tau^{L}\right) & \\
=1-\left(1-1 \times\left(\left(\frac{1}{\left(1-(1-u)^{\kappa}\right)^{\gamma}}\right.\right.\right. & \\
& \left.\left.\left.+\frac{1}{\left(1-(1-v)^{\kappa}\right)^{\gamma}}-1\right)^{1 / \gamma}\right)^{-1}\right)^{1 / \kappa},
\end{aligned}
$$

where $\kappa=1 / \log _{2}\left(2-\tau^{U}\right)$ and $\gamma=-1 / \log _{2} \tau^{L}$.
Tail dependence captures the behavior of the random variables during extreme events. The normal copula has $\tau^{U}=$ $\tau^{L}=0$ when correlation parameter is less than one, meaning that in the extreme tails of the distribution the variables are independent. The Joe-Clayton copula allows both upper and lower tail dependence to range from zero to one freely of each other. One major drawback of the Joe-Clayton copula is that even when the two tail dependence measures are equal, there is still some (slight) asymmetry. The symmetrized Joe-Clayton (SJC) copula has overcome this drawback by a slight modification of the original Joe-Clayton copula, which ensures that the tail dependence is symmetric when $\tau_{U}=\tau_{L}$.

For the time-varying form of SJC copula, Patton [18] suggested that the tail dependence coefficients follow something akin to a restricted ARMA $(1,10)$ process:

$$
\begin{aligned}
& \tau_{t}^{U}=\Lambda\left(\omega_{1}+\beta_{1} \tau_{t-1}^{U}+\alpha_{1} \cdot \frac{1}{10} \sum_{i=1}^{10}\left|u_{t-i}-v_{t-i}\right|\right) \\
& \tau_{t}^{L}=\Lambda\left(\omega_{2}+\beta_{2} \tau_{t-1}^{U}+\alpha_{2} \cdot \frac{1}{10} \sum_{i=1}^{10}\left|u_{t-i}-v_{t-i}\right|\right),
\end{aligned}
$$

where $\Lambda$ denotes the logistic transformation $\Lambda(x)=(1+$ $\left.e^{-x}\right)^{-1}$ so as to keep the parameters of SJC copula in $(0,1)$ and $u_{i}$ and $v_{i}$ come from the probability integral transform series $U$ and $V$ by using the marginal cumulative distribution function of the original series. The tail dependence evolution equation contains an autoregressive term and a forcing variable, which is the mean absolute difference between $u$ and $v$ over the previous 10 observations.

2.5. Estimation. This paper's main interest is to estimate the dependence parameters in copula functions. Maximum likelihood is the natural estimation procedure to use in this context that specifies models for the two marginal distributions and the copula. There are two approaches to estimate the parameters in a copula function using maximum likelihood estimation (MLE). The first and most direct estimation method is to estimate the copula and the marginal distributions simultaneously. But in this approach, the large number of parameters can make numerical maximization of the likelihood function difficult. The second approach is a two-stage maximum likelihood estimation method that the marginal distribution functions are estimated with the assumption of independence between the two random variables firstly and then the dependence parameter of copula function is estimated by substituting the marginal distribution into the copula function. In this paper, we apply the twostage maximum likelihood method.

\section{Empirical Analysis}

This section contains the empirical part of the paper. First, the data source is described. Afterwards, the ARMA-GARCH filtering of the data is sketched, which will ensure that the goodness-of-fit tests get i.i.d. data as input. Finally, we estimate the parameters of two time-varying copulas and generate the time-varying correlation coefficient of the 
loan growth and economy growth. We use the software of Eviews8.0 and Matlab2012 to finish the computation.

3.1. Data. In our empirical analysis, it includes two indices which are economy growth index and loan growth index. GDP is the most ideal variable of economy growth. But it is well known that GDP is a quarterly statistical index. To increase the sample point, we choose the growth rate of Chinese industrial added value, which is a monthly economic index, as the proxy of economy growth. In fact, the growth rate of GDP and industrial added value has highpositive correlation. The growth rate of industrial added value is gathered from the WIND database and the website of National Bureau of Statistics of China. The loan growth rate is gathered from the website of People's Bank of China and China Financial Statistics (1949-2005). The monthly data covers the period of January, 1992 to December, 2013. According to Chinese industrial statistics rules, the industrial added value of January has not been submitted from the year of 2007. We apply the average interpolation approach to complete the data series. Table 1 presents some summary statistics of the data.

3.2. Filtering of the Data. The copula model needs i.i.d. data as input. However, both series exhibit positive skewness. The Jarque-Bera test of each growth rate strongly rejects unconditional normality. Plots of the autocorrelation function and the partial autocorrelation function of the series and the squared series show that the time series exhibit autocorrelation and time-varying conditional volatility. These visual results are also confirmed by formal statistical tests, such as the LjungBox test, which rejects the null hypothesis of no autocorrelation, and Engle's Lagrange multiplier test, which indicates that there are indeed ARCH effects. To remove autocorrelation and conditional heteroscedasticity in the univariate time series of the growth rates, an ARMA model with GARCH errors is fitted to the raw series, respectively. We use the AIC, SIC, and Hannan-Quinn criteria to determine the optimal lag length for the conditional mean (ARMA) process. According to these criteria, we select $m=2$ and $n=1$ for the loan growth rate and $m=2$ and $n=2$ for the growth rate of industrial added value. The lags of GARCH model are supposed to equal one because a lot of literature has indicated that the GARCH model with one lag is usually sufficient to provide good estimation of the conditional volatility of most macroeconomic and financial variables (see, e.g., [23]). By comparing the outcome of the ARMA-GARCH model with different distribution, we find that the model with generalized error distribution (GED) can preferably fit the series. The estimated parameters and standard errors for marginal distribution models are presented in Table 2.

Applying the Ljung-Box test and Engle's LM test to the filtered series, both up to lag 10, the null hypothesis of no autocorrelation and no ARCH effects, respectively, cannot be rejected any more. Then, we transform the standardized error series to uniform distribution series $U$ and $V$ by using the cumulative distribution function of GED. The KolmogorovSmirnov $(K-S)$ test indicate the hypothesis that $U$ and $V$ are uniform $(0,1)$ and cannot be rejected.
TABLE 1: Summary statistics.

\begin{tabular}{lcc}
\hline & Loan growth rate & $\begin{array}{c}\text { Growth rate of industrial } \\
\text { added value }\end{array}$ \\
\hline Mean & 18.9048 & 14.0346 \\
Std. Dev. & 5.1818 & 4,3156 \\
Skewness & 0.7920 & 0.7027 \\
Kurtosis & 3.6216 & 3.7667 \\
Jarque-Bera stat & $28.4406^{*}$ & $29.0476^{*}$ \\
Ljung-Box stat & $1525.3^{*}$ & $876.77^{*}$ \\
ARCH LM stat & $3.5049^{*}$ & $2.3368^{*}$ \\
Linear correlation & \multicolumn{2}{|c}{0.2556} \\
\hline
\end{tabular}

This table presents some summary statistics of the data used in this paper. The Ljung-Box test and ARCH LM test are conducted using 10 lags. An asterisk $(*)$ indicates a rejection of the null hypothesis at the 0.05 level.

3.3. Result for the Copulas. Stock returns have been found to take on joint negative extremes more often than joint positive extremes, leading to the observation that "stocks tend to crash together but not boom together." No similar empirical evidence is yet available for the dependence relation of loan growth rate and economy growth rate. We will specify and estimate two alternative copulas, the symmetrized JoeClayton copula and the Gaussian copula. The symmetrized Joe-Clayton specification allows asymmetric dependence on the lower tail and upper tail. We now present the main results of this paper: the estimation results for the normal and symmetrized Joe-Clayton (SJC) copula models. For the purpose of comparison, we also present the results for these two copulas without time variation in the copula parameters. The estimated outcome of copula models is presented in Table 3.

For the static Gaussian copula, the correlation parameter is positive $(0.2677)$ and is statistically significant. For the time-varying Gaussian copula, the parameters $\alpha$ and $\beta$ are also statistically significant and satisfy the parameter constraints $\alpha+\beta<1$. The parameters of time-varying symmetrized Joe-Clayton copula are also significant. From the estimation outcome of static symmetrized Joe-Clayton copula, the upper tail dependence parameter $\tau^{U}$ is not significant, whereas the lower tail dependence parameter $\tau^{L}$ is statistically significant although it is a small number. This demonstrates that the loan and economy growth is positively correlated at the lower tail but is almost independent at the upper tail. By comparing the AIC, BIC, and LLF values, we find that the time-varying symmetrized Joe-Clayton copula that considers both the asymmetric correlation of the two tails and time-varying feature is the best fitting model. Whether it is a static or time-varying model, the symmetrized JoeClayton copula which considers the asymmetric tail correlation performs better than the Gaussian copula. Whether it is a Gaussian copula or SJC copula model, the time-varying type performs better than the static one.

The time path of the conditional correlations implied by the time-varying copula was obtained from simulations based on two marginal models and the estimated time-varying copula models (see [24]). Specifically, at each time point 
TABLE 2: Results for the marginal distributions.

\begin{tabular}{|c|c|c|c|c|c|}
\hline & & \multicolumn{2}{|c|}{ Loan growth rate } & \multicolumn{2}{|c|}{ Growth rate of industrial added value } \\
\hline & & Coefficient & Std error & Coefficient & Std error \\
\hline \multirow{5}{*}{ Mean equation } & $\mathrm{C}$ & 0.4973 & 0.2784 & 1.0235 & 0.1936 \\
\hline & $\operatorname{AR}(1)$ & 0.9592 & 0.0007 & 0.9141 & 0.0139 \\
\hline & $\operatorname{AR}(2)$ & -0.2363 & 0.0415 & -0.6548 & 0.0597 \\
\hline & MA(1) & 0.1654 & 0.0388 & -0.3216 & 0.1232 \\
\hline & $\mathrm{MA}(2)$ & - & - & -0.1592 & 0.0309 \\
\hline \multirow{3}{*}{ Variance equation } & $\mathrm{C}$ & 0.3678 & 0.1615 & 1.8259 & 0.9891 \\
\hline & $\mathrm{ARCH}$ & 0.5697 & 0.1762 & 0.4093 & 0.1691 \\
\hline & GARCH & 0.2728 & 0.1159 & 0.4365 & 0.1812 \\
\hline \multicolumn{2}{|c|}{ GED parameter } & 0.8001 & 0.0831 & 0.7872 & 0.0751 \\
\hline
\end{tabular}

TABLE 3: Results for the copula models.

\begin{tabular}{|c|c|c|c|c|c|c|}
\hline & & Coefficient & Std error & AIC & $\mathrm{BIC}$ & LLF \\
\hline Static Gaussian & $\rho$ & 0.2677 & 0.0651 & 16.3375 & 37.7704 & -2.169 \\
\hline \multirow{2}{*}{ Static SJC } & $\overline{\tau^{U}}$ & 0.0063 & 0.0087 & \multirow{2}{*}{12.9831} & \multirow{2}{*}{29.8561} & \multirow{2}{*}{-1.9983} \\
\hline & $\tau^{L}$ & 0.0453 & 0.0112 & & & \\
\hline \multirow{2}{*}{ Time-varying Gaussian } & $\alpha$ & 0.0166 & 0.0027 & \multirow{2}{*}{1.6456} & \multirow{2}{*}{13.9982} & \multirow{2}{*}{0.8956} \\
\hline & $\beta$ & 0.9044 & 0.0611 & & & \\
\hline \multirow{6}{*}{ Time-varying SJC } & Constant $^{U}$ & -8.2656 & 0.7290 & \multirow{6}{*}{0.8920} & \multirow{6}{*}{8.0363} & \multirow{6}{*}{1.554} \\
\hline & $\alpha^{U}$ & -5.7305 & 1.1402 & & & \\
\hline & $\beta^{U}$ & 2.5747 & 0.6055 & & & \\
\hline & Constant ${ }^{L}$ & -2.9160 & 0.0776 & & & \\
\hline & $\alpha^{L}$ & -0.5875 & 0.0183 & & & \\
\hline & $\beta^{L}$ & 4.2438 & 0.7775 & & & \\
\hline
\end{tabular}

$t(t=1,2, \ldots, 264)$, we generate 2000 pairs of bivariate uniform distribution variables $\left(u_{i, t}, v_{i, t}\right)(i=1,2, \ldots, 2000)$ from the time-varying copula model according to the estimated parameter. Then the simulated pairs of uniformly distributed $\left(u_{i, t}, v_{i, t}\right)$ were transformed into random variables $\left(x_{i, t}, y_{i, t}\right)$ according to the conditional marginal distribution implied by the estimated ARMA-GARCH-GED model. Then we can get the time-varying correlation estimation by computing the linear correlation coefficient of series $\left\{x_{i, t}\right\}$ and $\left\{y_{i, t}\right\}(i=$ $1,2, \ldots, 2000)$ at each time point $t$. For getting the confidence interval of the correlation coefficient, we generate 1000 correlation coefficient at each time point $t$ by repeating the above stochastic simulation process 1000 times. Then we can estimate the confidence interval at each time point $t$ by the simulated 1000 correlation coefficients.

Figure 1 illustrates the time path of conditional correlation parameter and GDP growth rate. The correlation parameter is ranging from 0.1776 to 0.4474 and the average is 0.3179 . These indicate that there is a positive correlation relationship between Chinese banking loan growth and economy growth. In other words, Chinese loan growth is procyclical, which means that loan growth commoved with the economy growth. But the dependency is not very strong partially because of the strong control ability of Chinese government in the financial sector. By carefully studying the evolution of dependence, we find that the procyclicality of
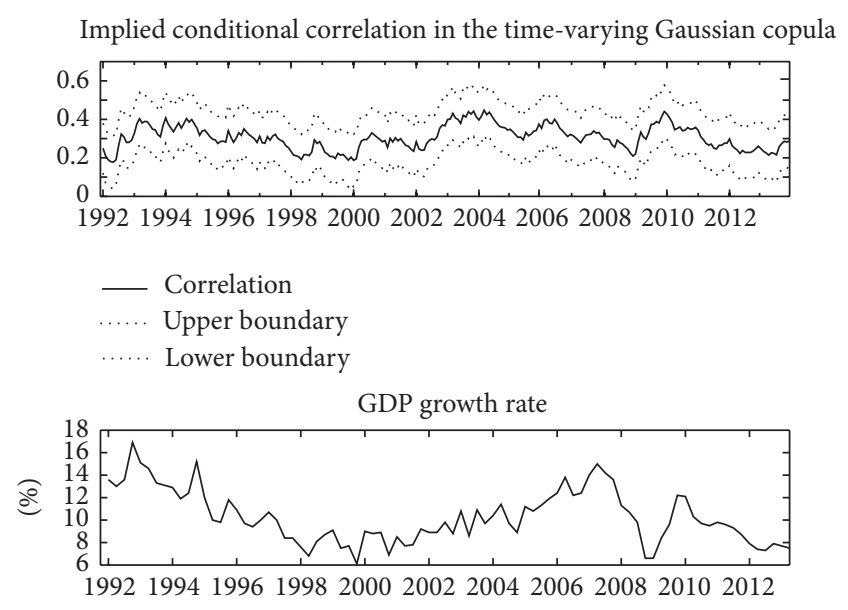

Figure 1: Conditional correlation in the time-varying Gaussian copula and Chinese GDP growth rate, with 95\% confidence interval for the time-varying correlation.

bank loan is varying with the economy cycle. When GDP growth rate goes down, the dependence of loan and economy will increase. Oppositely, when GDP growth rate goes up, the dependence of loan and economy will decrease. This means that the procyclicality of banking loan growth is much 

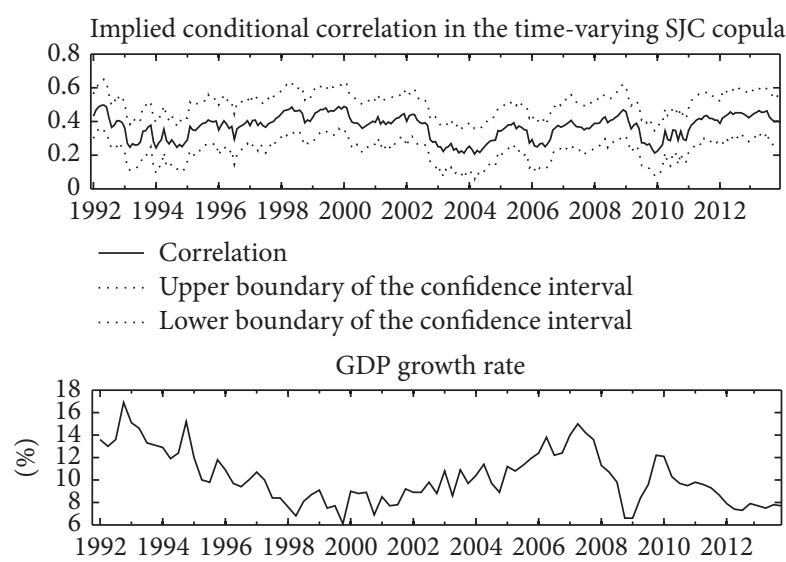

FIGURE 2: Implied conditional correlation in the time-varying SJC copula and Chinese GDP growth rate, with 95\% confidence interval for the time-varying correlation.

stronger in the decline stage than in the rise stage of the economy. Specifically, during the Asia financial crisis of 1997 and the subprime mortgage crisis in 2008, the dependence is obviously higher than other periods. Figure 2 illustrates the implied time path of the conditional dependence in the timevarying symmetrized Joe-Clayton copula and GDP growth rate. The plot is similar to that in Figure 1, and the negative correlation between dependent degree and GDP growth rate is again very clear.

Figure 3 illustrates the time path of conditional upper tail dependence, conditional lower tail dependence of the timevarying symmetrized Joe-Clayton copula and the difference of them. The conditional upper tail dependence is ranging from 0.0054 to 0.0203 and the average is 0.0108 . The standard error of the upper tail dependence parameter in the static SJC copula is 0.0087 . The $95 \%$ confidence interval of the upper tail dependence parameter contains 0 , so the conditional upper tail dependence is not significant. The conditional lower tail dependence is ranging from 0.0455 to 0.0601 and the average is 0.0524 . The $95 \%$ confidence interval of constant lower tail dependence parameter does not contain 0 , so the conditional lower tail dependence is significant. Furthermore, the difference between the lower and upper tail dependence of the time-varying symmetrized Joe-Clayton Copula is significantly above zero as illustrated in Figure 3. This means that the procyclicality of banking loan growth is much stronger during severe contractionary business cycles than the opposite.

\section{Results Analysis and Implications}

All the above indicate that the procyclicality of Chinese loan growth is asymmetrical. The dependence of loan and economy growth is stronger in the decline stage than in the rise stage of the economy. There are three probable reasons which induce this asymmetry.

First, the asymmetry of ratings' procyclicality during upward and downward period of business cycle may contribute to the asymmetric procyclicality of banking loan
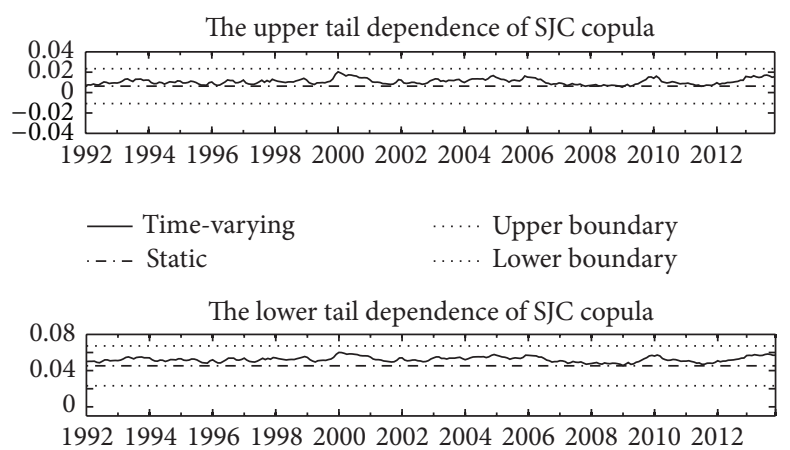

$$
\begin{array}{lll}
\text { _ Time-varying } & \text {...... Upper boundary } \\
\text {..- Static } & \text {..... L Lower boundary }
\end{array}
$$

The difference between the upper and the lower tail dependence of SJC copula

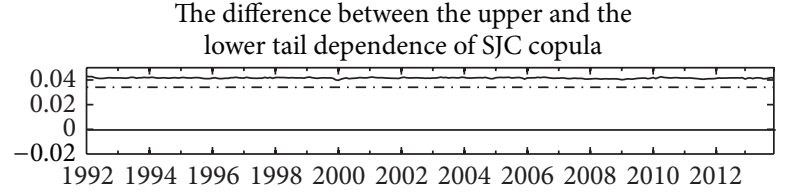

- Time-varying

-. - Static

FIGURE 3: The conditional upper and lower tail dependence in timevarying symmetrized Joe-Clayton copula, with $95 \%$ confidence interval for the constant correlation case.

growth. As discussed by Amato and Furfine [25], the credit rating agencies tend to make more downgrades when there are negative changes in the rated companies' financial position which is significantly influenced by the economic cycle. In contrast, during the upward phase of the cycle, the ratings improve asymmetrically because the rating agencies are backward-looking rather than forward-looking in their assessments. This asymmetric procyclicality of ratings can induce the asymmetric procyclicality of loan by the credit supply channel. During a crisis, capital requirements for banks will significantly rise since firms of borrowing from bank may be downgraded, thus further sharply reducing credit supply. On the contrary, the increase of credit supply is not symmetrical.

Second, asymmetric monetary policy effects can contribute to the asymmetric behavior of loan growth. Bliss and Kaufman [26] argued that it may be easier for the central bank to restrain booms than to stimulate recoveries because capital requirements are more likely to become binding at the bottom of a business cycle. Kishan and Opiela [27] also argued that expansionary policy is not effective in stimulating the loan growth of low-capital banks.

Third, the possible asymmetry of bank managers' herd behavior may also induce the asymmetry of banking procyclicality. Rajan [28] related that herd behavior is an inherent cause of banking procyclicality. In fact, some researchers argued that the herd behavior is asymmetric, where the investor is relatively cautious to chase in the rising stage and is panic to escape in the collapse stage of stock price. Ribeiro and Veronesi [29] argued that risk-averse investors with uncertainty about the state of the world can be shown 
to generate such a dependent structure. This asymmetric herd behavior may also exist in the bank managers due to the well-known principal-agent problem. The bank managers try to avoid jeopardizing their position if they follow wrong personal choices. They tend to tighten the credit approval because they should take the responsibility of loan loss in the decline stage of economy when this risk will rise.

Coming back to the issue of the decision of taking countercyclical buffer, our research results combined with the previous results of other researchers may partly explain the disfunction of credit-to-GDP gap for the decision of releasing buffer. First, as illustrated by Repullo and Saurina [30], credit usually lags the business cycle, especially in downturns, and the use of deviations of the credit-to-GDP ratio with respect to its trend compounds the problem, because it takes some time before the ratio crosses the trend line. For example, during the recent crisis real credit growth even increased initially in several countries, such as the UK and Spain [12]. Second, according to our findings, the procyclicality of banking loan is stronger in the decline stage than in the rise stage of economy. This means that, in case the trend of loan growth turns around, comparing with the rise stage, the decline of loan growth with the economy is more rapid and dramatic. Because of the above two reasons, the credit-toGDP gap often cannot give accurate signal for releasing the buffer capital.

The lag of signalling for releasing countercyclical buffer is crucial in that the supply of credit may be constrained by regulatory capital requirements in downturns, which can contribute to the further recess of macroeconomy and the subsequent banking instability. Actually, the Basel Committee was aware of the shortcomings of the credit-to-GDP gap, in particular in downturns, and proposed to use supervisory judgment to release the buffer. The committee also suggested that promptly releasing the buffer in times of stress can help to reduce the risk of the supply of credit being constrained by regulatory capital requirements. But how to realize promptly releasing the buffer still is a question waiting for answer. We give two rough answers. First, the authority can use high frequent index as the releasing indicator for timely capturing the turn point. For example, the monthly macroeconomic index such as industrial added value can be used for signalling the start point of releasing. Of course, the selection for proper high frequent index still needs serious study in the future. Second, during the downturn of economy, the authority should accelerate the pace of releasing the buffer compared with building it up in the rise stage of economy. The authority can introduce a releasing adjustment factor, which is larger than one and the value is determined by the asymmetric degree of procyclicality, to accelerate the releasing pace.

\section{Conclusions}

This paper applies copula modeling methods in order to study the characteristic of Chinese banking procyclicality. We mainly analyze the existence of asymmetric procyclicality of loan growth by utilizing time-varying copula to Chinese data from January 1992 to December 2013. Standard ARMAGARCH models with GED innovations are employed for the marginal distributions of the growth rate of loan and industrial added value to filter the data. Then the bivariate Gaussian copula and the symmetrized Joe-Clayton copula, which allows for general asymmetric dependence, were estimated. The time-varying dependence structure is captured by allowing the parameters of the two copulas to comply with an evolution equation.

The empirical research demonstrates that the procyclicality of loan growth is asymmetric. The dependence between loan and economy growth is more correlated in the decline stage than in the rise stage of economy. The asymmetric credit ratings change may induce loan growth's asymmetric procyclical behavior by the credit supply channel. And also, the asymmetric monetary policy effect and the asymmetric herd behavior of bank managers may contribute to the asymmetric procyclicality.

We conclude that the asymmetric procyclicality and the lag of credit can explain the disfunction of credit-to-GDP gap for the decision of releasing phase in taking countercyclical buffer of Basel III. The lag of signalling for releasing countercyclical buffer is crucial in that the supply of credit may be constrained by regulatory capital requirements in downturns, which can contribute to the further recess of macroeconomy and the subsequent banking instability. We suggest two rough thoughts for promptly releasing the buffer. First, the authority can use high frequent index as the releasing indicator for timely signalling the release start point. Second, during the downturn of economy, the authority should accelerate the pace of releasing countercyclical buffer compared with building it up in the rise stage of economy.

\section{Conflict of Interests}

The authors declare that there is no conflict of interests regarding the publication of this paper.

\section{Acknowledgments}

The authors thank Andrew Patton who kindly provided the MATLAB code of Patton_copula_toolbox on his home page http://public.econ.duke.edu/ ap172/. This research is supported by National Natural Science Foundation of China under grant 71231008 .

\section{References}

[1] J. M. Keynes, The General Theory of Employment, Interest and Money, McMillan, London, UK, 1936.

[2] B. S. Bernanke and A. S. Blinder, "Credit, money, and aggregate demand," American Economic Review Papers and Proceedings, vol. 78, no. 2, pp. 435-439, 1989.

[3] N. Kiyotaki and J. Moore, "Credit cycles," Journal of Political Economy, vol. 105, no. 2, pp. 211-248, 1997.

[4] Bank for International Settlements (BIS), 80th Annual Report, Basel, Switzerland, 2010, http://www.bis.org.

[5] T. Jokipii and A. Milne, "The cyclical behaviour of European bank capital buffers," Journal of Banking \& Finance, vol. 32, no. 8, pp. 1440-1451, 2008. 
[6] M. Puri, J. Rocholl, and S. Steffen, "Global retail lending in the aftermath of the US financial crisis: distinguishing between supply and demand effects," Journal of Financial Economics, vol. 100, no. 3, pp. 556-578, 2011.

[7] A. N. Berger, R. DeYoung, M. Flannery, D. Lee, and O. Oztekin, "How do large banking organizations manage their capital ratios?" in Federal Reserve Bank of Kansas City Research Working Paper, 2008.

[8] European Central Bank (ECB), "Special features-determinants of bank lending standards and the impact of financial turmoil," Financial Stability Review, pp. 135-140, 2009.

[9] Bank of Greece, “Monetary Policy 2009-2010," 2010.

[10] P. P. Athanasoglou, I. Daniilidis, and M. D. Delis, "Bank procyclicality and output: issues and policies," Journal of Economics and Business, vol. 72, pp. 58-83, 2014.

[11] BCBS, Basel III: A Global Regulatory Framework for More Resilient Banks and Banking Systems, Basel Committee on Banking Supervision, 2011.

[12] BCBS, Guidance for National Authorities Operating the Countercyclical Capital, Basel Committee on Banking Supervision, 2010.

[13] M. Drehmann, C. Borio, L. Gambacorta, G. Jimenez, and C. Trucharte, "Countercyclical capital buffers: exploring options," BIS Working Paper, 2010.

[14] J. Marcucci and M. Quagliariello, "Asymmetric effects of the business cycle on bank credit risk," Journal of Banking \& Finance, vol. 33, no. 9, pp. 1624-1635, 2009.

[15] A. J. Patton, "A review of copula models for economic time series," Journal of Multivariate Analysis, vol. 110, pp. 4-18, 2012.

[16] B. V. M. Mendes, "Asymmetric extreme interdependence in emerging equity markets," Applied Stochastic Models in Business and Industry, vol. 21, no. 6, pp. 483-498, 2005.

[17] F. Durante and P. Jaworski, "Spatial contagion between financial markets: a copula-based approach," Applied Stochastic Models in Business and Industry, vol. 26, no. 5, pp. 551-564, 2010.

[18] A. J. Patton, "Modelling asymmetric exchange rate dependence," International Economic Review, vol. 47, no. 2, pp. 527$556,2006$.

[19] T. Bollerslev, "Generalized autoregressive conditional heteroskedasticity," Journal of Econometrics, vol. 31, no. 3, pp. 307327, 1986.

[20] A. Sklar, "Fonctions de repartition à $\mathrm{n}$ dimensions et leurs marges," Publications de l'Institut Statistique de l'Universite de Paris, vol. 8, pp. 229-231, 1959.

[21] R. B. Nelsen, An Introduction to Copulas, Springer Series in Statistics, Springer, New York, NY, USA, 2nd edition, 2006.

[22] R. Engle, "Dynamic conditional correlation: a simple class of multivariate generalized autoregressive conditional heteroskedasticity models," Journal of Business \& Economic Statistics, vol. 20, no. 3, pp. 339-350, 2002.

[23] T. Bollerslev, R. Y. Chou, and K. F. Kroner, "ARCH modeling in finance: a review of the theory and empirical evidence," Journal of Econometrics, vol. 52, no. 1-2, pp. 5-59, 1992.

[24] K. Wang, Y.-H. Chen, and S.-W. Huang, “The dynamic dependence between the Chinese market and other international stock markets: a time-varying copula approach," International Review of Economics and Finance, vol. 20, no. 4, pp. 654-664, 2011.

[25] J. D. Amato and C. H. Furfine, "Are credit ratings procyclical?" BIS Working Papers 129, 2003.
[26] R. R. Bliss and G. G. Kaufman, "Bank procyclicality, credit crunches, and asymmetric monetary policy effects: a unifying model," Journal of Applied Finance, vol. 13, no. 2, pp. 23-21, 2003.

[27] R. P. Kishan and T. P. Opiela, "Bank capital and loan asymmetry in the transmission of monetary policy," Journal of Banking \& Finance, vol. 30, no. 1, pp. 259-285, 2006.

[28] R. Rajan, "Has financial development made the world riskier?" in Proceedings of the Federal Reserve Bank of Kansas City Economic Symposium at Jackson Hole, 2005.

[29] R. Ribeiro and P. Veronesi, "The excess comovement of international stock returns in bad times: a rational expectations equilibrium model," Working Paper, Graduate School of Business, University of Chicago, 2002.

[30] R. Repullo and J. Saurina, "The countercyclical capital buffer of Basel III: a critical assessment," CEPR Discussion Paper Series 8304, 2011. 


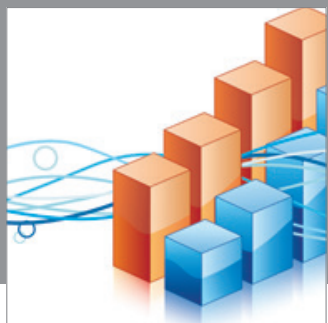

Advances in

Operations Research

mansans

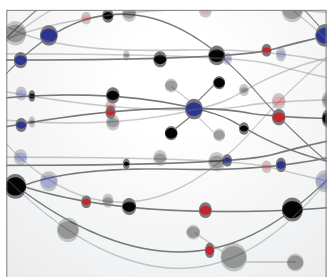

The Scientific World Journal
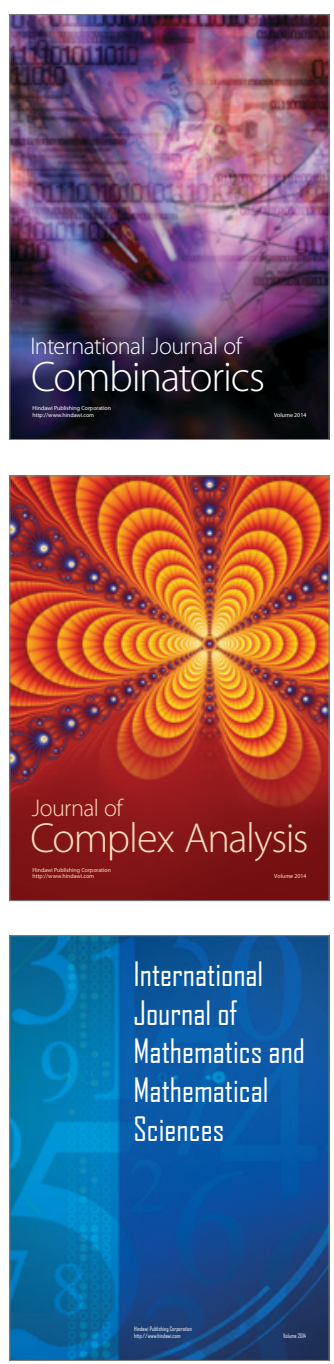
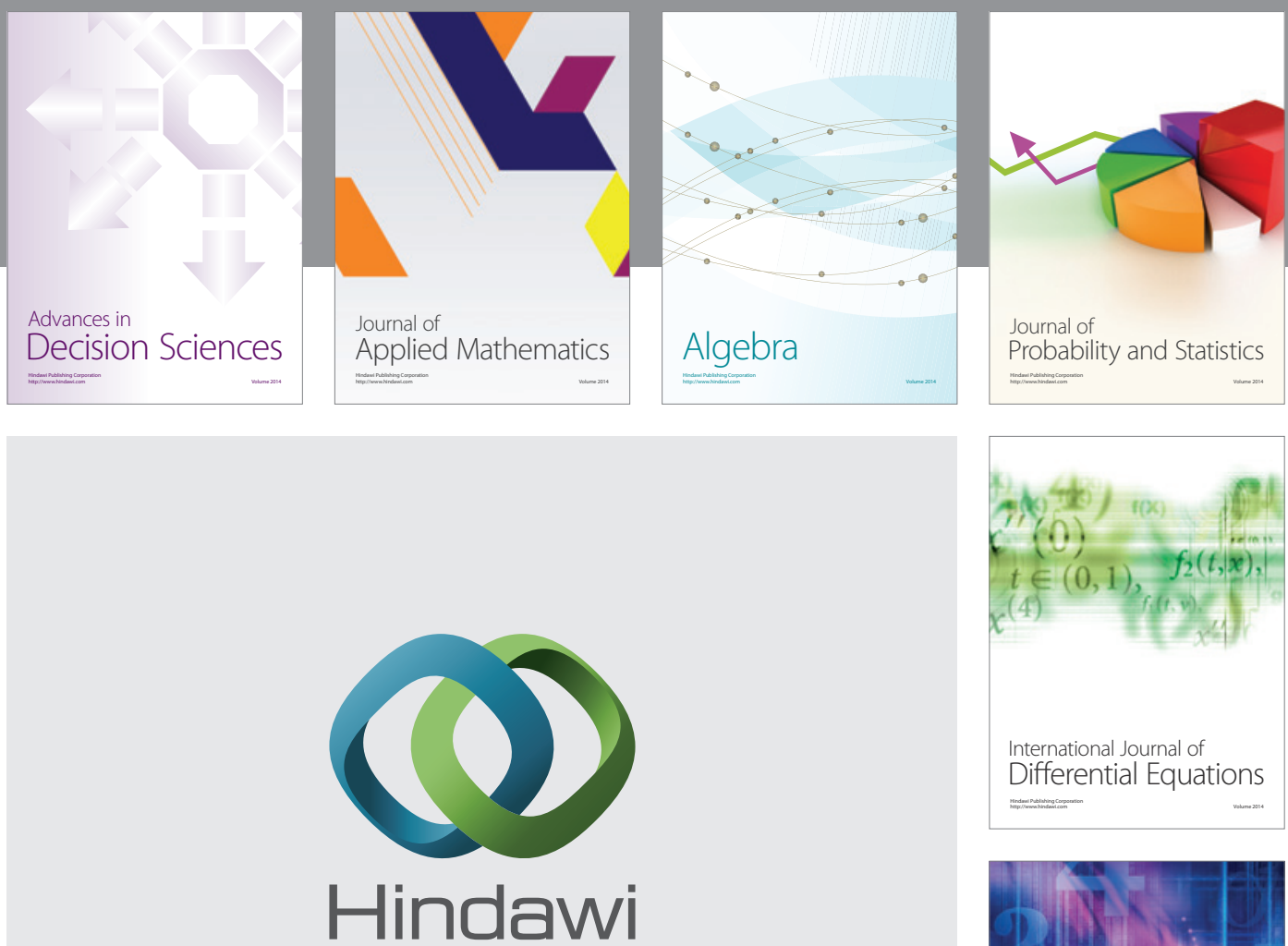

Submit your manuscripts at http://www.hindawi.com
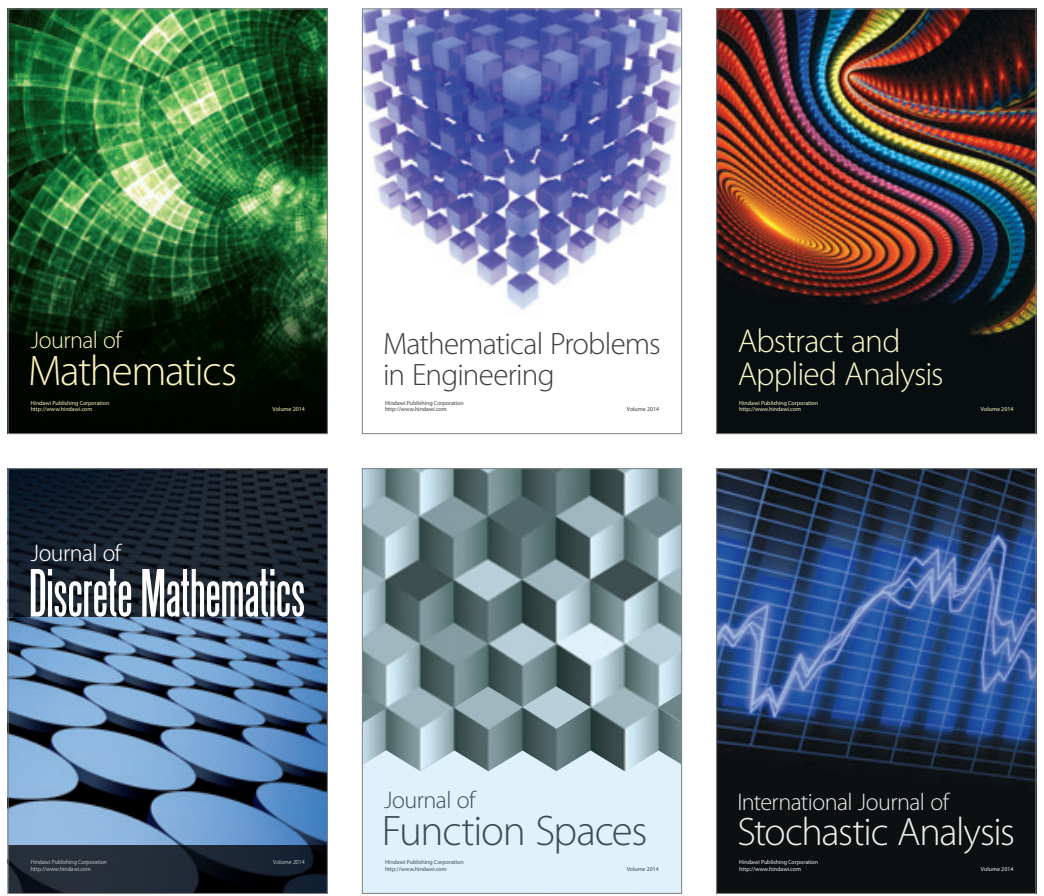

Journal of

Function Spaces

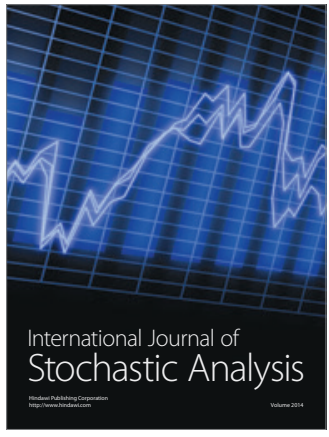

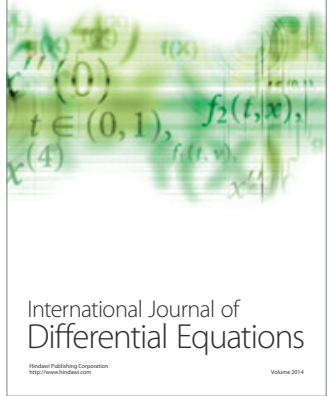
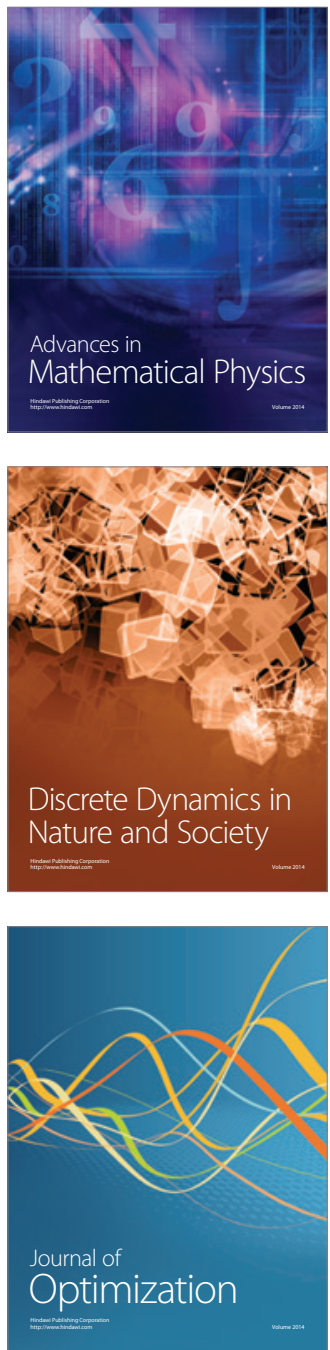